\title{
Renormalization in momentum dependent resummations
}

\author{
Antal Jakovád \\ Institute of Physics, Budapest University of Technology and Economics, Budafoki út 8, H-1111 Budapest , Hungary
}

(Dated: August 29, 2018)

\begin{abstract}
At finite temperature and in non-equilibrium environments we have to resum perturbation theory to avoid infrared divergences. Since resummation shuffles the perturbative orders, renormalizability is a nontrivial issue. In this paper we demonstrate that one can modify any type of resummations - even if it is momentum dependent, or it resums higher point functions - in a way that it is renormalizable with the usual zero temperature counterterms. To achieve this goal we reformulate resummation in form of a renormalization scheme. We apply this technique to perform a $2 \mathrm{PI}$ resummation in the six dimensional cubic scalar model in equilibrium.
\end{abstract}

PACS numbers: 11.10Wx, 11.10Gh, 11.15Tk

\section{INTRODUCTION}

Renormalized perturbation theory in $\overline{\mathrm{MS}}$ scheme was developed to extract perturbative information about vacuum expectation values of observables at high energies. Still, when it fails, for example in computation of expectation values with a generic density matrix, we tend to speak about "non-perturbative effects" and the failure of perturbation theory (PT). An example at high temperature when this pathological behavior occurs was demonstrated by Dolan and Jackiw 1], showing that in $\Phi^{4}$ theory at finite temperatures higher order diagrams can provide contributions $\sim\left(\lambda T^{2} / m^{2}\right)^{n}$ which is large at high temperatures. They also found the solution of this problem: with (re)summation of a specific set of diagrams (the daisy diagrams), the problematic $\sim \lambda T^{2}$ term becomes part of the propagator mass, thus appearing in the denominator instead of the numerator.

This solution reveals, at the same time, the core of the problem. Perturbation theory in $\overline{\mathrm{MS}}$ scheme starts with a free action, fitted to the zero temperature particle spectrum. At finite temperatures the true excitation spectrum changes significantly. Since particle spectrum comes from the free part of the Lagrangian, any perturbation theory will fail which tries to follow this change via interactions. This line of thought also provides an easier way of performing daisy resummation, by changing particle spectrum of the theory from the beginning. Technically one adds to and subtracts from the Lagrangian the same (temperature dependent) mass term, treating one as a part of the free action, the other as a (thermal) counterterm [2]. The modification mass term can be set equal to the high temperature limit of the self energy diagram, or it can be optimized via a gap equation $[3,4]$.

Taking into account thermal effects only through an effective mass can be used only cautiously in case of more complicated situations, as in non-equilibrium, or in gauge theories [21]. In these cases the non-trivial, non-quadratic momentum dependence of the self energy is important, too, and it must be taken into account already in the free Lagrangian. In gauge theories the hard thermal loop (HTL) improved perturbation theory [5] provides this type of resummation, which uses the high temperature limit of the momentum dependent self energy as part of the effective propagator (and uses also effective vertices to maintain gauge invariance, cf. [5]). In the generic case the modification of the spectrum can be taken into account most effectively with the method of Cornwall, Jackiw and Tomboulis $\underline{6}$, which performs two-particle-irreducible (2PI) resummation. In this method we should compute the diagrams with the exact (also background field dependent) propagator, and, consequently, no self-energy corrections should be taken into account. This method was intensively developed and used in numerical calculations in the recent literature [7].

The consequent application of all these methods is obstructed by their non-trivial relation to the renormalization. Direct application of the thermal counterterm method, for example, requires temperature dependent infinite counterterms to cancel the one-loop level $\sim m_{T}^{2} / \varepsilon$ type of divergences, where $m_{T}^{2}$ is the thermal mass [4, 8, 9]. The temperature dependent piece drops out at two loop level, but there new temperature dependent divergences are generated. The situation is even more serious in the case of 2PI method: at one loop level we generate terms to the exact propagator that are $\sim m^{2} \log p$; when putting back into loops, this term provides us with new types of divergences, not present in the original renormalized Lagrangian. Therefore the resummation obtained by truncating the 2PI equations at a given loop order is not consistent as a renormalizable theory.

*Electronic address: Antal.Jakovac@cern.ch 
The main reason of failure is that we separate the loop order of the IR sensitive diagram we want to resum and the corresponding counterterm diagrams, which, in the original PT, made them finite. In a resummation based on fixed order skeleton diagrams this leads to uncompensated divergences. There are different methods to keep track of the divergences. The local case is simpler and there we have a deeper understanding of the problem. A selfconsistent method for resummation was developed in [8, 10] to obtain finite gap equations. In [1] the self-consistent resummation in the framework of $2 \mathrm{PPI}$ effective action is accomplished by explicitely introduced counterterms into the Lagrangian. In [9] this method is generalized to any, momentum-independent resummations. Here an alternative viewpoint is applied: the resummation being treated as a change of renormalization scheme. The idea of adapting the renormalization process to the environment was first suggested in [12].

The momentum dependent resummations are more difficult, here the results are concentrated on the 2PI resummation. In a seminal paper, van Hees and Knoll [13] presented a proof based on the BPHZ scheme showing that it is in fact enough to use the zero temperature BPHZ scheme to renormalize the finite temperature 2PI resummation. The central problem there is to renormalize the zero temperature ladder resummation, ie. the Bethe-Salpeter equations. This method was further developed and used in [14, 15, 16]. A method to renormalize Hartree-Fock approximation was developed in 17].

It is, however, still an open question, what kind of momentum dependent resummations are renormalizable. In this paper we study this question, primarily in the framework of the four dimensional $\Phi^{4}$ and six dimensional $\Phi^{3}$ model, but the logic can be easily extended to more complicated models. The starting point is the method of [9], which is generalized to momentum dependent case. The main idea is that, instead of resumming the necessary counterterm diagrams in the original resummation, we slightly change the resummation procedure: in the low energy (IR) regime it will be still the desired resummation, while at high energies (UV) it falls back to the ordinary perturbation theory. This means that the divergences remain identical to the usual zero temperature ones.

The paper is structured as follows. In Section II we give a different view of resummation, interpreting it as a specific renormalization scheme even in the momentum dependent case. We generalize somewhat the notion of "renormalized Lagrangian", allowing momentum dependent parameters, and taking care not to spoil the perturbative renormalizability. In Section III we summarize the results of 2PI resummation, and find that specific resummation scheme that corresponds to it. It will be shown that it is not a well-defined scheme (as it can be conjectured from the renormalization problems of the 2PI resummation itself), so in Section $\amalg$ IIB we suggest improvements of the method that is capable to satisfy renormalizability and 2PI resummation in the IR regime at the same time. In Section IV we apply the method to find the $2 \mathrm{PI}$ resummed self energy of the six dimensional $\Phi^{3}$ model. In Section $\nabla$ we give our concluding remarks and discuss open questions.

\section{REINTERPRETING RESUMMATION}

Resummation is a notion that pre-assumes a singled out perturbation theory (the "unresummed" one). It suggests that in this singled out perturbation theory (PT) we have to do additional work to compute some observables by taking into account contributions from higher orders. Usually this singled out PT is the one stemming from the $\overline{\mathrm{MS}}$ renormalized Lagrangian. This works nicely when used for calculation of vacuum expectation values of high energy observables, but needs to be resummed, for example, at finite temperatures.

This way of thinking is, however, misleading, as we have no a priori reason to prefer one environment/process above others. Having in mind this democracy of environments, we should not speak about resummation, but about perturbation theories best adapted to a certain environment (cf. also 12]).

The definition of quantum field theory is based on the path integral living on a fine enough spacetime lattice, with an action (the bare action) that have parameters depending on the lattice spacing. This UV action provides, in principle, the expectation values of any IR observables, at any environment. If we do PT, however, we must have some knowledge about the result in the specific environment in order that already the first approximation is "sufficiently" close to the exact result: ie. we should ensure that the corrections are indeed "perturbative", smaller than the leading order. Since in the usual PT the solvable part is the free (quadratic) theory, the above requirement says that the vacuum as well as the free excitation spectrum should be sufficiently close to the exact one. But the vacuum of the theory may change in different phases of the matter, and also the excitation spectrum can be dramatically different at different physical environments, for example at different temperatures, not to speak about the non-equilibrium environment. So, although it is implicit in the usual discussions, before we start PT, half of the task should be solved: to guess the vacuum and the spectrum sufficiently well.

Different PTs, adapted to different environments, yield different results already at tree level. But, of course, at infinite order any consistent PT should provide the same result for a given observable. That means that the result of the tree level of PT $A$ is somehow hidden in the higher orders of PT $B$. From the point of view of $B$, therefore, $A$ represents a resummation of the perturbative series - and vice versa. And both $A$ and $B$ are resummations of the 
bare perturbative series. A zero temperature example is that results obtained in the on-mass shell scheme seem to be "resummed" from the point of view of the $\overline{\mathrm{MS}}$ scheme.

In addition to the necessity of resummation there is another problem. When we start with a UV theory with appropriate bare parameters, it is granted that any IR observables are "finite", ie. do not depend on the exact value of the UV cutoff. The requirement for a consistent PT is, however, much more severe: we should have finite results order by order. Only a very smart resummation of the bare theory, known as the renormalized PT, can provide finite results at each perturbative order [18]. No other perturbative methods are known that would be capable to render the theory IR finite. Therefore, if renormalizability is an issue, we must not use other PTs than the renormalized ones.

Technically, in renormalized PT, we split the parameters into a renormalized and counterterm part, the latter being cutoff dependent, and should formally be taken into account at higher orders of the calculation. Renormalizability requires specific form only for the UV part. Thus it allows to work with different PTs, which use different finite parts of the counterterms: these are called renormalization schemes (RSs). When we want to fit the PT to the external environment the only thing we can play with is the finite part of the counterterms; ie. we should adapt the RS to the environment by choosing appropriate finite parts.

\section{A. Class of constant physics}

In the family of RSs we can define an equivalence relation: two RSs are equivalent if they provide the same expectation values for any $n$-point functions at infinite order (up to a finite normalization factor). This leads to the equivalence classes of schemes that provides "constant physics".

Let us take two RSs: $A$ with parameters $g_{i}^{A}$, and $B$ with parameters $g_{i}^{B}$. The expectation value of an $n$-point function $\hat{O}_{n}=\mathrm{T} \Phi\left(x_{1}\right) \ldots \Phi\left(x_{n}\right)$ will depend on these parameters:

$$
\left\langle\hat{O}_{n}\right\rangle^{A}=O_{n}^{A}\left(g^{A}\right), \quad\left\langle\hat{O}_{n}\right\rangle^{B}=O_{n}^{B}\left(g^{B}\right) .
$$

$A$ and $B$ belong to the same class of constant physics, if we have

$$
O_{n}^{A}\left(g^{A}\right)=\zeta_{A B}^{n} O_{n}^{B}\left(g^{B}\right),
$$

with a ( $n$-independent) constant $\zeta_{A B}$. According to the theory of renormalization [18] these equations can be fulfilled for any $n$, if there is a specific relation between the renormalized parameters

$$
g_{i}^{A}=f_{i}^{A B}\left(g^{B}\right) .
$$

Sometimes these equations are referred to as the renormalization group (RG) equations. The physical condition for providing the same values for the observables from both schemes is that they both stem from the same bare Lagrangian [18].

To demonstrate these notions we give an example in the $\Phi^{4}$ model. The Lagrangian reads in terms of the bare quantities (denoted by subscript "0"):

$$
\mathcal{L}=\frac{1}{2}\left(\partial \Phi_{0}\right)^{2}-\frac{m_{0}^{2}}{2} \Phi_{0}^{2}-\frac{\lambda_{0}}{24} \Phi_{0}^{4} .
$$

The renormalized Lagrangians are used to write in the following form

$$
\mathcal{L}=\frac{1}{2}(\partial \Phi)^{2}-\frac{m^{2}}{2} \Phi^{2}-\frac{\lambda}{24} \Phi^{4}+\frac{\delta Z^{2}}{2}(\partial \Phi)^{2}-\frac{\delta m^{2}}{2} \Phi^{2}-\frac{\delta \lambda}{24} \Phi^{4}
$$

where the formal power of $\lambda$ in the counterterms determine the loop order they first contribute. Since (4) determines, nonperturbatively, the expectation values of all IR observables, clearly the renormalized Lagrangians of (5) are giving the same value (at infinite order), if we satisfy

$$
\Phi_{0}=Z \Phi, \quad Z^{2} m_{0}^{2}=m^{2}+\delta m^{2}, \quad Z^{4} \lambda_{0}=\lambda+\delta \lambda,
$$

where $Z^{2}=1+\delta Z^{2}$. Therefore two schemes give the same result (up to normalization), if they obey:

$$
Z_{A}=\zeta_{A B} Z_{B}, \quad m_{A}^{2}+\delta m_{A}^{2}=\zeta_{A B}^{2}\left(m_{B}^{2}+\delta m_{B}^{2}\right), \quad \lambda_{A}+\delta \lambda_{A}=\zeta_{A B}^{4}\left(\lambda_{B}+\delta \lambda_{B}\right) .
$$


The condition that schemes $A$ and $B$ belong to the same class of constant physics can be rewritten compactly, using the generator of 1PI diagrams as

$$
\Gamma_{A}[\Phi]=\Gamma_{B}\left[\zeta_{A B} \Phi\right]
$$

At finite $n$th order this is satisfied up to order $g^{n}$. Then the left hand side contains contributions from higher orders, as considered from RS $B$. So the left hand side provides a resummation for RS $B$. Moreover, since all the functions $f^{A B}$ and $O^{A}$ are finite, we have a finite, ie. renormalized resummation.

\section{B. Momentum independent resummations}

Let us try to apply the ideas of the previous subsection to the momentum independent mass resummation in $\Phi^{4}$ model. A more comprehensive study of this case can be found in Ref. [9].

Let us choose for $\mathrm{RS} A$ the $\overline{\mathrm{MS}}$ scheme. At one loop level with dimensional regularization the counterterms are defined as [9]:

$$
\delta Z_{\overline{\mathrm{MS}}, 1}=0, \quad \delta m_{\overline{\mathrm{MS}}, 1}^{2}=-m^{2} \frac{\lambda}{32 \pi^{2}}\left[-\frac{1}{\varepsilon}+\gamma_{E}-1-\ln 4 \pi\right], \quad \delta \lambda_{\overline{\mathrm{MS}}, 1}=-\frac{3 \lambda^{2}}{32 \pi^{2}}\left(-\frac{1}{\varepsilon}+\gamma_{E}-\ln 4 \pi\right) .
$$

In this scheme zero temperature observables can be calculated with reasonable precision; at finite temperatures, however, IR divergences make the calculation difficult. For example the 1-loop self energy at zero momentum reads, in dimensional regularization:

$$
\Pi_{\overline{\mathrm{MS}}}(p=0)=m^{2}+\frac{\lambda m^{2}}{32 \pi^{2}}\left[-\frac{1}{\varepsilon}+\gamma_{E}-1+\ln \frac{m^{2}}{4 \pi \mu^{2}}\right]+\frac{\lambda}{2} J(m)+\delta m_{\overline{\mathrm{MS}}, 1}^{2}
$$

where $\mu$ is the renormalization scale. $J$ is the finite temperature part of the bosonic tadpole function:

$$
J(m)=\frac{1}{2 \pi^{2}} \int_{m}^{\infty} d \omega \sqrt{\omega^{2}-m^{2}} n(\omega)
$$

where $n(\omega)$ is the Bose-Einstein distribution. It can be approximated at high temperatures as

$$
J(m) \approx \frac{T^{2}}{12}-\frac{T m}{4 \pi}-\frac{m^{2}}{16 \pi^{2}} \ln \frac{m^{2}}{c T^{2}}+\ldots,
$$

where $\ln c=1+2 \ln 4 \pi-2 \gamma_{E} \approx 4.90762$. Therefore, at high temperatures, the one-loop self energy will be

$$
\Pi_{\overline{\mathrm{MS}}}(p=0, T)=m^{2}+\frac{\lambda T^{2}}{24}-\frac{\lambda T m}{8 \pi}+\frac{\lambda m^{2}}{32 \pi^{2}} \ln \frac{c T^{2}}{4 \pi \mu^{2}} .
$$

This means that the first order correction can be larger than the tree level term, if $\lambda T^{2} \gg m^{2}$, ie. at high temperatures. At higher orders this failure of the $\overline{\mathrm{MS}}$ scheme becomes more and more obvious, as we receive $\left(\lambda T^{2} / m^{2}\right)^{n}$ contributions from the daisy diagrams [1].

This phenomenon, however, does not mean the failure of perturbation theory itself, since we can easily find a RS, where this problem does not show up. Indeed, the finite temperature version of the on-mass-shell (M) scheme uses the counterterm

$$
\delta m_{M 1}^{2}=-\frac{\lambda m^{2}}{32 \pi^{2}}\left[-\frac{1}{\varepsilon}+\gamma_{E}-1+\ln \frac{m^{2}}{4 \pi \mu^{2}}\right]-\frac{\lambda}{2} J(m)
$$

and so the self energy in this scheme reads simply

$$
\Pi_{M}(p=0, T)=m^{2}
$$

This is just the tree level value - as we expect from the on-mass-shell scheme. No dangerous $\lambda T^{2}$ terms appear, and by definition it extends to all orders in PT. So it seems to be much better to use the on-mass-shell scheme at high temperatures for perturbative calculations. 
If we want to compare the results of the $\overline{\mathrm{MS}}$ and the finite temperature on-mass-shell schemes - for example if we want to follow the temperature dependence of the quantities starting from zero temperature - then we must take care that they belong to the same class of constant physics. Since now only the mass counterterm is changed, we have $\zeta=1$ and $\lambda_{\overline{\mathrm{MS}}}=\lambda_{M}=\lambda$, and we must satisfy

$$
m_{\overline{\mathrm{MS}}}^{2}-\frac{\lambda m_{\overline{\mathrm{MS}}}^{2}}{32 \pi^{2}}\left[-\frac{1}{\varepsilon}+\gamma_{E}-1-\ln 4 \pi\right]+\mathcal{O}\left(\lambda^{2}\right)=m_{M}^{2}-\frac{\lambda m_{M}^{2}}{32 \pi^{2}}\left[-\frac{1}{\varepsilon}+\gamma_{E}-1+\ln \frac{m_{M}^{2}}{4 \pi \mu^{2}}\right]-\frac{\lambda}{2} J\left(m_{M}\right)+\mathcal{O}\left(\lambda^{2}\right) .
$$

At $\mathcal{O}(\lambda)$ this simply yields

$$
m_{\overline{\mathrm{MS}}}^{2}=m_{M}^{2}-\frac{\lambda m_{M}^{2}}{32 \pi^{2}} \ln \frac{m_{M}^{2}}{\mu^{2}}-\frac{\lambda}{2} J\left(m_{M}\right) .
$$

When we solve this equation - a finite gap equation - the result will be applicable in the given class of constant physics. In particular, we can interpret this result as a resummed $\overline{\mathrm{MS}}$ self energy:

$$
\Pi \frac{\text { resummed }}{\mathrm{MS}}(p=0, T)=m_{M}^{2}\left(m_{\overline{\mathrm{MS}}}, \lambda\right) .
$$

An alternative to this approach is when we define a scheme where only the dangerous finite temperature part is resummed. This $F$ scheme is defined by the counterterm

$$
\delta m_{F 1}^{2}=-\frac{\lambda m^{2}}{32 \pi^{2}}\left[-\frac{1}{\varepsilon}+\gamma_{E}-1-\ln 4 \pi\right]-\frac{\lambda}{2} J(m)
$$

and so the self energy in this scheme reads simply

$$
\Pi_{F}(p=0, T)=m^{2}\left[1+\frac{\lambda}{32 \pi^{2}} \ln \frac{m^{2}}{\mu^{2}}\right] .
$$

This is the zero temperature $\overline{\mathrm{MS}}$ value, and we avoided the dangerous $\lambda T^{2}$ terms again. In this case the condition of the constant physics reads:

$$
m_{\overline{\mathrm{MS}}}^{2}-\frac{\lambda m_{\overline{\mathrm{MS}}}^{2}}{32 \pi^{2}}\left[-\frac{1}{\varepsilon}+\gamma_{E}-1-\ln 4 \pi\right]+\mathcal{O}\left(\lambda^{2}\right)=m_{F}^{2}-\frac{\lambda m_{F}^{2}}{32 \pi^{2}}\left[-\frac{1}{\varepsilon}+\gamma_{E}-1-\ln 4 \pi\right]-\frac{\lambda}{2} J\left(m_{F}\right)+\mathcal{O}\left(\lambda^{2}\right),
$$

which, at $\mathcal{O}(\lambda)$, implies

$$
m_{\overline{\mathrm{MS}}}^{2}=m_{F}^{2}-\frac{\lambda}{2} J\left(m_{F}\right)
$$

This is another, also usual, form of the gap equation. The resummation again reads as

$$
\Pi_{\mathrm{MS}}^{\text {resummed }}(p=0, T)=\left.\Pi_{F}(p=0, T)\right|_{m_{F}\left(m_{\overline{\mathrm{MS}}}, \lambda\right)} .
$$

This is a finite version of the thermal mass method.

\section{Momentum dependent schemes}

What was said above is perfectly applicable to momentum independent resummations, as it was demonstrated in Ref. [9]. To be able to treat momentum dependence we have to rethink what are really the conditions for a Lagrangian to be renormalizable.

In fact, all theorems of renormalizability are based on the behavior of the Feynman diagrams when internal momenta are asymptotically large. One should prove that a diagram can be divergent if some of its internal momenta go to infinity on the same rate [18]; we do not have new divergences when different momenta go to infinity at different rate. One proves that after removing the divergences of all sub-diagrams the diagram is superficially divergent with polynomial momentum dependence. Then the BPHZ scheme and forest formula go through, and we can perturbatively renormalize the theory [18]. 
It is clear that all these theorems still hold, if propagators or vertices are momentum dependent, but in a way that the asymptotic behavior is the same as in the usual case. More precisely, the new momentum dependence should not cause any new divergences in any diagrams.

For example, if we want to use momentum dependent "mass" term in $\Phi^{4}$ theory, we have to take into account, that the most singular diagram contains one propagator and one integration (the tadpole). If the mass has asymptotic momentum dependence as

$$
m^{2}(p)=m_{R}^{2}+\mathcal{O}\left(p^{-\gamma}\right),
$$

then the divergence of the tadpole diagram calculated with this "mass" reads

$$
\int \frac{d^{4} p}{(2 \pi)^{4}} \frac{1}{p^{2}-m^{2}(p)}=\int \frac{d^{4} p}{(2 \pi)^{4}} \frac{1}{p^{2}-m_{R}^{2}-\mathcal{O}\left(p^{-\gamma}\right)} \longrightarrow \int \frac{d^{4} p}{(2 \pi)^{4}}\left[\frac{1}{p^{2}-m_{R}^{2}}+\mathcal{O}\left(\frac{p^{-\gamma}}{\left(p^{2}-m_{R}^{2}\right)^{2}}\right)\right] .
$$

Here the second term yield finite result if $\gamma>0$; then it is not relevant for the issue of renormalizability. As a consequence we are allowed to use any momentum dependent mass term that approaches constant value polynomially at asymptotic large momenta.

This means that we can define a consistent renormalization scheme, by splitting the bare mass term as

$$
Z m_{0}^{2}=m^{2}(p)+\delta m^{2}(p)
$$

where $m^{2}(p)$ satisfies (24).

If the coefficient of the quadratic term is considered, ie. $p^{2}-m^{2}$, then the allowed modification is $\mathcal{O}\left(p^{-2-\gamma}\right)$ relative to the leading $p^{2}$ term. A similar observation can be made for coefficients of higher powers. For example, if the quadratic coupling is denoted as $\lambda$, then we can introduce a momentum dependent coupling as

$$
\frac{1}{24} Z^{2} \lambda_{\text {bare }}(2 \pi)^{4} \delta\left(\Sigma p_{i}\right) \Phi\left(p_{1}\right) \Phi\left(p_{2}\right) \Phi\left(p_{3}\right) \Phi\left(p_{4}\right)=\frac{1}{24}\left(\lambda\left(p_{i}\right)+\delta \lambda\left(p_{i}\right)\right)(2 \pi)^{4} \delta\left(\Sigma p_{i}\right) \Phi\left(p_{1}\right) \Phi\left(p_{2}\right) \Phi\left(p_{3}\right) \Phi\left(p_{4}\right) .
$$

If at high momenta the asymptotic behavior of $\lambda_{R}$ is denoted as

$$
\lambda\left(p_{1}, p_{2}, p_{3}, p_{4}\right)=\lambda_{R}+\mathcal{O}\left(\left(\max p_{i}\right)^{-2-\gamma}\right),
$$

then the tadpole diagram is computed as

$$
\int \frac{d^{4} p}{(2 \pi)^{4}} \frac{\lambda(p, q, k, \ell)}{p^{2}-m^{2}(p)} \longrightarrow \int \frac{d^{4} p}{(2 \pi)^{4}}\left[\frac{\lambda_{R}}{p^{2}-m_{R}^{2}}+\mathcal{O}\left(\frac{p^{-2-\gamma}}{p^{2}-m_{R}^{2}}\right)\right]
$$

where the second term is convergent if $\gamma>0$.

So as a general conclusion we can state that a Lagrangian remains perturbatively renormalizable if the couplings are momentum dependent, provided they are suppressed by a factor $\mathcal{O}\left(p^{-2-\gamma}\right)$ relative to the leading momentum power at high momenta.

Finally we give two remarks. First, the order $\mathcal{O}\left(p^{-2-\gamma}\right)$ claims nothing about logarithmic correction, since it has sub-power asymptotics. So, for example, an asymptotic behavior $\log (p) p^{-2-\gamma}$ is still allowed. The second remark is that we may not stop at the level of the usual renormalizable operators. We can add $\sim \Phi^{6}$ terms with coefficient of the leading term $\mathcal{O}\left(p^{-2-\gamma}\right)$. This makes the renormalizable treatment of nPI diagrams possible.

\section{Momentum dependent renormalizable resummations}

Now we are ready to generally describe the resummations that are renormalizable at the same time. We restrict ourselves here to self-energy type resummations.

We first define a scheme (M) that is particularly adequate for the problem at hand. In this case it is simply the choice of the momentum dependent mass counterterm $\delta m_{M}^{2}(p)$. Anticipating matching to $\overline{\mathrm{MS}}$ scheme, we also use momentum dependent mass $m^{2}(p)$. To simplify the notation we introduce the renormalized free propagator in this scheme as

$$
G_{M, 0}^{-1}(p)=p^{2}-m^{2}(p) .
$$

Now we perform perturbation theory in this scheme. Since it is a well defined scheme, all expectation values are finite. In particular the 1PI generating functional $\Gamma_{M}[\Phi]$ is finite, too. This will depend on the propagator; to emphasize this dependence we shall write $\Gamma_{M}\left[\Phi ; G_{M, 0}\right]$. 
Now we translate the results to $\overline{\mathrm{MS}}$ scheme. Since only the masses are modified (that is $\delta Z^{2}$ and $\delta \lambda$ are the same as in $\overline{\mathrm{MS}}$ ), the condition of the constant physics reads simply:

$$
m_{\overline{\mathrm{MS}}}^{2}+\delta m_{\overline{\mathrm{MS}}}^{2}=m_{M}^{2}(p)+\delta m_{M}^{2}(p)
$$

In practical cases $\delta m_{M}^{2}(p)$ depends on the propagator $G_{M, 0}(p)$, and so this is a gap equation. Since the left hand side is momentum independent, $m_{M}^{2}(p)$ should compensate the momentum dependence of $\delta m_{M}^{2}(p)$. If the counterterm has a polynomially vanishing momentum dependence, the mass will also have polynomially vanishing momentum dependence. In this case, according to the analysis of the previous subsection, the divergences in $\delta m_{\overline{M S}}^{2}$ and $\delta m_{M}^{2}(p)$ are the same. That means, that we have a finite relation between $m_{M}^{2}(p)$ and $m_{\overline{M S}}^{2}-$ or, expressing in another way, the propagator in scheme $M$ depends explicitly on the $\overline{\mathrm{MS}}$ mass value: $G_{M, 0}\left(p ; m_{\overline{\mathrm{MS}}}\right)$.

With this dependence all expectation values are the same in $\overline{\mathrm{MS}}$ and in $M$ schemes, if we calculated them at infinite order. At finite $n$th order they are the same only up to $\lambda^{n}$, the rest is the "resummation" as considered from the point of view of $\overline{\mathrm{MS}}$ scheme:

$$
\Gamma_{\overline{\mathrm{MS}}}^{\text {resummed }}[\Phi]=\left.\Gamma_{M}\left[\Phi ; G_{M, 0}\right]\right|_{G_{M, 0} \text { from constant physics }}
$$

To make this, in essence very simple, strategy more tractable, we now work out some examples in the next sections.

\section{RENORMALIZATION OF 2PI RESUMMATION}

A popular resummation method is the 2PI resummation; for a comprehensive description and further references cf. [6, 7]. The physical idea is that the free theory should characterize the quasiparticle propagation in the best possible way, so we should use the exact propagator already in the free theory. Therefore in perturbation theory the internal lines are exact propagators, which also implies that it must not contain self-energy insertions. Since selfenergy insertions are separated from the rest of the diagram by two lines, the above idea is based on diagrams that are two-particle irreducible. In the usual formalism it is achieved by introducing a generic propagator as an external quadratic current, and then perform a Legendre transformation on that. The requirement that the free propagator is exact can be formulated as a gap equation.

There are different methods to renormalize 2PI resummation; these usually use BPHZ scheme, and rely on the renormalization of the zero temperature Bethe-Salpeter kernel [13]. The method proposed by this paper makes the 2PI effective action finite in a different way, directly using the (generalized) counterterms of the original Lagrangian.

\section{A. The 2PI resummation as a renormalization scheme}

In the skeleton diagrams of the $2 \mathrm{PI}$ resummation the self-energy diagrams are missing. We can define a scheme, where this occurs, by appropriately choosing the mass counterterm. To understand the idea we recall the zero temperature on-mass shell scheme: here one cancels radiative mass corrections on the mass shell with help of the finite part of the mass counterterm, thus achieving that the tree level mass is the exact mass in the same time. The exact mass reads as

$$
m_{e x}^{2}=m^{2}+\delta m^{2}+\left.\Pi(k)\right|_{k^{2}=m_{e x}^{2}}
$$

and we have to ensure that it agrees with the free mass, ie. $m_{e x}^{2}=m^{2}$. That yields the following choice of the mass counterterm

$$
\delta m^{2}+\left.\Pi(k)\right|_{k^{2}=m^{2}}=0,
$$

therefore the renormalized self-energy correction is zero on the mass shell. To fully define the scheme, we should choose the finite parts of the other counterterms $(\delta \lambda, \delta Z)$ : we choose for them the $\overline{\mathrm{MS}}$ values.

We should generalize this scheme in a way that not only the mass, but the complete exact propagator is the same as the free propagator. We use a momentum dependent mass $m(k)$, yielding the free propagator $G^{-1}=k^{2}-m^{2}(k)$, together with momentum dependent mass counterterm $\delta m(k)$. From Schwinger-Dyson equation, using explicitly the mass counterterm, we can write

$$
G_{e x}^{-1}(k)=G^{-1}(k)-G_{e x}^{-1}(k)\left(\Pi(k)+\delta m^{2}(k)\right) G^{-1}(k) .
$$


The condition that the free propagator is exact, $G_{e x}(k)=G(k)$, can be satisfied for all $k$ if

$$
\delta m^{2}(k)+\Pi(k)=0 .
$$

This is in direct analogy with the on-mass-shell equation (34). We will call the scheme defined above as bare 2PI scheme.

Two remarks are in order here. First we call the attention to the fact that $\Pi(k)$ implicitly depends on the propagator $G$ so on the momentum dependent mass $m(k)$, too. Secondly, if the bare 2PI scheme was a consistent scheme (as it is not, see later), the above condition should determine the finite part only, the regularized UV divergences should be the same as in any other schemes.

If we want to have the result in $\overline{\mathrm{MS}}$ scheme we must ensure that the $2 \mathrm{PI}$ and the $\overline{\mathrm{MS}}$ schemes belong to the same class of constant physics. According to our previous analysis in Section $\amalg \mathrm{C}$ we should provide the same the bare mass in the two cases:

$$
Z^{-2}\left(m^{2}(k)+\delta m^{2}(k)\right)=Z \frac{-2}{\mathrm{MS}}\left(m_{\overline{\mathrm{MS}}}^{2}+\delta m_{\overline{\mathrm{MS}}}^{2}\right) .
$$

We can choose the same wave function renormalization counterterm in the two schemes. Then, using (36), we can write

$$
m^{2}(k)-\Pi[m, k]=m_{\overline{\mathrm{MS}}}^{2}+\delta m_{\overline{\mathrm{MS}}}^{2},
$$

where we explicitly written out the mass dependence of the self-energy. Equivalently we can write

$$
m^{2}(k)=m_{\overline{\mathrm{MS}}}^{2}+\Pi_{\overline{\mathrm{MS}}}[m, k] .
$$

Here $\Pi_{\overline{\mathrm{MS}}}[m, k]=\Pi[m, k]-\delta m_{\overline{\mathrm{MS}}}^{2}$, a finite quantity (if the bare $2 \mathrm{PI}$ scheme was a consistent renormalization scheme). If we add $\mathrm{a}-k^{2}$ to both sides we find

$$
G^{-1}(k)=G_{\overline{\mathrm{MS}}}^{-1}-\Pi_{\overline{\mathrm{MS}}}[G, k] .
$$

This equation is exactly the same as the $2 \mathrm{PI}$ gap equation that determines the self energy, or, equivalently, $G(k)$.

\section{B. Improvement of the $2 \mathrm{PI}$ resummation}

Although the formalism of the 2PI resummation is appealing, it is inconsistent when using order by order. The self energy, namely, can yield corrections of type $k^{2} \ln k, m^{2} \ln k$, which, if considered to be part of the propagator, leads to new, eventually temperature dependent, divergences in the calculation that cannot be canceled by usual counterterms. The related problem in the bare 2PI scheme is that the condition (39) requires that $m^{2}(k)$ contains momentum dependence $\sim k^{2} \ln k$ or $\sim m^{2} \ln k$, which is not the allowed $\mathcal{O}\left(k^{-\gamma}\right)$ order. This means that the bare 2PI scheme is, in fact, not a consistent renormalization scheme.

In the present framework we can accommodate to the requirement of renormalizability, if we employ 2PI resummation in the $I R$ regime, and leave the asymptotic momentum regime untouched. These schemes are already consistent, and we call them 2PI schemes. There are several possibilities to achieve this.

The first is that we take into account the momentum dependence only below a scale $M$; above this scale we stay with the above discussed thermal mass approximation. That is we write for the mass counterterm, in high temperature approximation (cf. (14)):

$$
\delta m^{2}(k)= \begin{cases}-\Pi(k) & \text { if }|k|<M \\ \delta m_{\overline{\mathrm{MS}}}^{2}-\frac{\lambda T^{2}}{24}, & \text { if }|k|>M\end{cases}
$$

Since at asymptotically high momenta we recover Lorentz invariance, we can use for $|k|$ the Euclidean norm. The condition of the constant physics (39) now reads as

$$
m^{2}(k)=m_{\overline{\mathrm{MS}}}^{2}+\Theta(|k|<M) \Pi_{\overline{\mathrm{MS}}}[m, k]+\Theta(|k|>M)\left[\frac{\lambda}{2} J\left(m^{2}\right)+\delta m_{\overline{\mathrm{MS}}}^{2}\right] .
$$

This describes a scheme that provides 2PI resummation at low momenta, and super-daisy resummation at high momenta. Since we expect that the effects of the environment, and so the spectrum modifications are restricted to the IR regime, this choice hopefully catches the main physical points. 
We can apply, instead of a sharp cutoff, a smeared cutoff function $M(k)$ that is 1 for small momenta and vanishes asymptotically as $k^{-2-\gamma}$ at least. Then we could write

$$
\delta m^{2}(k)=-M(k) \Pi(k)-(1-M(k)) \frac{\lambda}{2} J\left(m^{2}\right),
$$

and the condition of the constant physics reads

$$
m^{2}(k)=m_{\overline{\mathrm{MS}}}^{2}+M(k) \Pi_{\overline{\mathrm{MS}}}[m, k]+(1-M(k))\left[\frac{\lambda}{2} J\left(m^{2}\right)+\delta m_{\overline{\mathrm{MS}}}^{2}\right] .
$$

Another possibility, related to the technique applied by [14], is to subtract the problematic part in the asymptotically large momentum region:

$$
\delta m^{2}(k)=-\Pi[m, k]+\Pi_{0}^{a s y m p t}\left[m_{R}, k\right] .
$$

The last symbol means the momentum dependence of the renormalized (in any scheme) vacuum self energy for asymptotically large momenta with mass $m_{R}$; so this is an explicit function of $m_{R}$ and $k$. If condition (24) is fulfilled, this equation ensures that the mass counterterm goes to a constant value at large momenta, since the momentum dependence in $\delta m^{2}(k)$ must be slower than that of any functions present in $\Pi_{\frac{\text { asympt }}{\mathrm{MS}}}\left[m_{R}, k\right]$. The condition of the constant physics reads

$$
m^{2}(k)=m_{\overline{\mathrm{MS}}}^{2}+\Pi_{\overline{\mathrm{MS}}}[m, k]-\Pi_{0}^{a s y m p t}\left[m_{R}, k\right] .
$$

Here again (24) is a self-consistent Ansatz: if it is obeyed by $m^{2}(k)$, then the right hand side goes to a constant value at high momenta faster than any functions in $\Pi_{0}^{a s y m p t}\left[m_{R}, k\right]$.

It must be emphasized that in any of the methods above we do not have complete 2PI resummation. To describe the high momentum regime we still have to rely on perturbation theory; but this regime is insensitive to matter effects and so any renormalization scheme applicable at zero temperatures is applicable here, too.

We still have the task to characterize the asymptotically large momentum regime. Let us consider a two-point function in a renormalized theory and go to this regime. Here the only dimensionful quantity is the momentum, which is made dimensionless with help of the renormalization scale. So we can write the self energy as

$$
\Sigma^{\text {(asym) }}(k) \approx k^{2} Z^{(\text {asym })}(k / \mu)+m^{2} Z_{m}^{\text {(asym) }}(k / \mu) .
$$

Therefore the dangerous terms in the asymptotic regime can be obtained from the scale dependence of the selfenergy. This means that we should count with logarithmic dependence, and the coefficients of the logarithms are the renormalization group beta-functions.

\section{2PI SCHEME IN SIX DIMENSIONAL $\Phi^{3}$ THEORY}

The simplest theory where we can demonstrate how the $2 \mathrm{PI}$ scheme introduced above works, is the $6 \mathrm{D} \Phi^{3}$ theory at finite temperature. The Lagrangian of the theory is written as

$$
\mathcal{L}=\frac{1}{2}(\partial \Phi)^{2}-\frac{m^{2}}{2} \Phi^{2}-\frac{g}{6} \Phi^{3}+\text { counterterms }
$$

Although the theory is not well defined in the path integral sense, it behaves well in the perturbation theory, and, moreover, it shows considerable resemblance with the perturbation theory of the gauge models. We will use one loop level resummation; we will have momentum dependence already at this level, so a momentum dependent resummation is needed. On the other hand the divergence structure is rather simple, which makes the treatment easy.

The most important peculiarity of the present treatment is that we allow momentum dependence in the mass counterterm, which implies momentum dependent mass. So the free Lagrangian can be written in momentum space

$$
\mathcal{L}=\frac{1}{2}\left(k^{2}-m^{2}(k)\right) \Phi(k) \Phi(-k)-\frac{1}{2} \delta m^{2}(k) \Phi(k) \Phi(-k),
$$

in such a way that the bare mass is untouched

$$
m_{\text {bare }}^{2}=m^{2}(k)+\delta m^{2}(k)=m_{\overline{\mathrm{MS}}}^{2}+\delta m_{\overline{\mathrm{MS}}}^{2} .
$$


We will use real time formalism in R/A basis [19]. Here the original fields $\left(\Phi_{1}, \Phi_{2}\right)$ are replaced by $\left(\Phi_{r}, \Phi_{a}\right)$ via the definition

$$
\Phi_{1}=\Phi_{r}+\frac{1}{2} \Phi_{a}, \quad \Phi_{2}=\Phi_{r}-\frac{1}{2} \Phi_{a}
$$

The propagator is a $2 \times 2$ matrix, where, in this basis $G_{a a}=0$ for the exact Green's function, and also $G_{r a}^{*}(k)=G_{a r}(k)$ is true in the Fourier space. The interaction Lagrangian reads

$$
-\mathcal{L}_{I}=\frac{g}{6}\left[\Phi_{1}^{3}-\Phi_{2}^{3}\right]=\frac{g}{2} \Phi_{r}^{2} \Phi_{a}+\frac{g}{24} \Phi_{a}^{3} .
$$

In the momentum dependent resummation we should use the most generic possible mass matrix. Since we are in equilibrium, all the propagators can be derived from the retarded propagator. This implies relations between the generic self energies, which we should respect when we choose the mass matrix. As it was pointed out in [20], and summarized in Appendix \$ the self-energies satisfy

$$
\begin{aligned}
& \operatorname{Re} \Sigma_{11}=-\operatorname{Re} \Sigma_{22}=\operatorname{Re} \Sigma_{R}, \quad \operatorname{Im} \Sigma_{11}=\operatorname{Im} \Sigma_{22}=\left(1+2 n_{B}\right) \operatorname{Im} \Sigma_{R}, \\
& \Sigma_{12}=-2 i n_{B} \operatorname{Im} \Sigma_{R}, \quad \Sigma_{21}=-2 i\left(1+n_{B}\right) \operatorname{Im} \Sigma_{R} .
\end{aligned}
$$

Here we used the notation $\Sigma_{R}=\Sigma_{a r}$ which is the retarded self energy. This is because it parameterizes the retarded Green's function as

$$
G_{R}^{-1}(k)=G_{R, 0}^{-1}(k)-\Sigma_{R}(k) .
$$

Choosing the mass matrix according to (53), the only independent parameter is the $m_{R}^{2}(k)$ retarded mass, and the free retarded propagator reads $G_{R, 0}^{-1}(k)=k^{2}-m_{R}^{2}(k)$. This implies for the free spectral function

$$
\varrho_{0}(k)=\operatorname{Disc}_{k_{0}} G_{R, 0}(k)=-2 \operatorname{Im} G_{R, 0}(k)=\frac{-2 \operatorname{Im} m_{R}^{2}(k)}{\left(k^{2}-\operatorname{Re} m_{R}^{2}(k)\right)^{2}+\left(\operatorname{Im} m_{R}^{2}(k)\right)^{2}} .
$$

From the properties of $\varrho_{0}(k)$ coming from causality, this equation requires that $\operatorname{Im} m_{R}^{2}(k)$ must be an odd function of $k_{0}$, and also $\operatorname{Im} m_{R}^{2}(k)<0$ must be true for $k_{0}>0$. In the $\overline{\mathrm{MS}}$ scheme it is satisfied by choosing $\operatorname{Im} m_{R}^{2}(k)=-\varepsilon \operatorname{sgn} k_{0}$.

Since $G_{R, 0}$ should be causal, $m_{R}^{2}$ must be either local or causal, ie. Kramers-Kronig relation holds true for it:

$$
m_{R}^{2}\left(k_{0}, \mathbf{k}\right)=\int_{-\infty}^{\infty} \frac{d \omega}{\pi} \frac{-\operatorname{Im} m_{R}^{2}(\omega, \mathbf{k})}{k_{0}-\omega+i \varepsilon}=\int_{0}^{\infty} \frac{d \omega}{\pi} \frac{2 \omega\left(-\operatorname{Im} m_{R}^{2}(\omega, \mathbf{k})\right)}{\left(k_{0}+i \varepsilon\right)^{2}-\omega^{2}} .
$$

Therefore $\operatorname{Im} m_{R}^{2}$ and the local parts of $m_{R}^{2}$ will completely determine $G_{R, 0}$.

For the counterterms we know that the infinite part is fixed, namely

$$
\mathcal{L}_{c t, d i v}=\frac{1}{2}\left(k^{2} \delta Z^{2}-\delta m_{\text {div }}^{2}\right)\left(\Phi_{1}^{2}-\Phi_{2}^{2}\right)-\frac{\delta g}{6}\left(\Phi_{1}^{2}-\Phi_{2}^{2}\right)^{2} .
$$

This implies $\operatorname{Re} \delta m_{R}^{2}=\delta m_{d i v}^{2}+$ finite, and $\operatorname{Im} \delta m_{R}^{2}=$ finite. We choose for $\delta Z^{2}$ and $\delta g$ their $\overline{\mathrm{MS}}$ values.

If we do perturbation theory in a generic scheme, all the diagrams are expressed through the propagators. Since all the propagators are functionals of $\varrho$, thus every diagram is a functional of $\varrho$. In particular, the one loop retarded self-energy in $\Phi^{3}$ model can be written as

$$
\Sigma_{R}(k)=g^{2} I_{R}(k)+\delta m_{R}^{2}(k)+k^{2} \delta Z^{2},
$$

where $I$ is the "bubble" diagram

$$
i I_{R}(k)=\int \frac{d^{6} p}{(2 \pi)^{6}} i G_{r r, 0}(p) i G_{r a, 0}(k-p) .
$$

At finite temperature the KMS relation implies

$$
i G_{r r, 0}(p)=\left(\frac{1}{2}+n\left(p_{0}\right)\right) \varrho_{0}(p) .
$$


Thus the imaginary part of the retarded self-energy reads

$$
\operatorname{Im} I_{R}(k)=-\frac{1}{2} \int \frac{d^{6} p}{(2 \pi)^{6}}\left(\frac{1}{2}+n\left(p_{0}\right)\right) \varrho_{0}(p) \varrho_{0}(k-p),
$$

indeed a functional of $\varrho$ alone.

For later convenience, this contribution can be divided into three parts, a "regular", a "singular" and a "divergent" part:

$$
I_{R}(k)=I_{R}^{\mathrm{div}}(k)+I_{R}^{\mathrm{sing}}(k)+I_{R}^{\mathrm{reg}}(k) .
$$

The divergent piece is defined that in a certain regularization this part goes to infinity as the regularization parameter vanishes. The singular part is defined as a contribution that grows at large momenta. Since the bubble contains no divergent sub-diagrams, these contributions can be calculated from the zero temperature result. In dimensional regularization this reads as

$$
I_{R}^{T=0}(k)=\left.\frac{1}{2(4 \pi)^{3}}\left[\left(\frac{1}{\varepsilon}-\gamma_{E}+1+\ln 4 \pi\right)\left(-\frac{k^{2}}{6}+m^{2}\right)-\int_{0}^{1} d x\left(-k^{2} x(1-x)+m^{2}\right) \ln \frac{-k^{2} x(1-x)+m^{2}}{\mu^{2}}\right]\right|_{k_{0} \rightarrow k_{0}+i \varepsilon}
$$

In the real part we have to take the modulus of the argument of the logarithm. The integral can be evaluated, the result reads

$$
\begin{aligned}
& \operatorname{Re} I_{R}^{T=0}(k)=\frac{1}{2(4 \pi)^{3}}\left[\left(\frac{1}{\varepsilon}-\gamma_{E}+\frac{8}{3}-\ln \frac{m^{2}}{4 \pi \mu^{2}}\right)\left(-\frac{k^{2}}{6}+m^{2}\right)-\frac{m^{2}}{3}+\frac{k^{2} \Gamma^{3}}{6} \ln \left|\frac{1+\Gamma}{1-\Gamma}\right|\right], \\
& \operatorname{Im} I_{R}^{T=0}(k)=-\Theta\left(k^{2}-4 m^{2}\right) \frac{\operatorname{sgn}\left(k_{0}\right)}{128 \pi^{2}} \frac{k^{2} \Gamma^{3}}{6},
\end{aligned}
$$

where

$$
\Gamma=\sqrt{1-\frac{4 m^{2}}{k^{2}}}
$$

What is particularly interesting is its large $k_{0}$ behavior; for a fixed $\mathbf{k}$ it can be obtained as the large $k$ behavior:

$$
\begin{aligned}
& \operatorname{Re} I_{R}^{T=0}(k \rightarrow \infty)=\frac{1}{2(4 \pi)^{3}}\left[\left(\frac{1}{\varepsilon}-\gamma_{E}+\frac{8}{3}-\ln \frac{k^{2}}{4 \pi \mu^{2}}\right)\left(-\frac{k^{2}}{6}+m^{2}\right)-\frac{m^{2}}{2}\right], \\
& \operatorname{Im} I_{R}^{T=0}(k \rightarrow \infty)=\frac{\operatorname{sgn}\left(k_{0}\right)}{128 \pi^{2}}\left(-\frac{k^{2}}{6}+m^{2}\right) .
\end{aligned}
$$

For the divergent pieces we choose the following expression

$$
g^{2} I_{R}^{\mathrm{div}}(k)=-k^{2} \delta Z_{0}^{2}-m^{2} \delta Z_{m}^{2},
$$

where

$$
\delta Z_{0}^{2}=\frac{1}{12(4 \pi)^{3}}\left(\frac{1}{\varepsilon}-\gamma_{E}+\frac{8}{3}+\ln 4 \pi\right), \quad \delta Z_{m}^{2}=-\frac{1}{2(4 \pi)^{3}}\left(\frac{1}{\varepsilon}-\gamma_{E}+\frac{13}{6}+\ln 4 \pi\right) .
$$

As a singular part we choose:

$$
I_{R}^{\operatorname{sing}}(k)=\left.\frac{1}{2(4 \pi)^{3}}\left(\frac{k^{2}}{6}-m^{2}\right) \ln \frac{M^{2}-k^{2}}{\mu^{2}}\right|_{k_{0} \rightarrow k_{0}+i \varepsilon}
$$

where $M^{2}$ is an arbitrary scale.

The regular piece, from (62), can be obtained as $I_{R}^{\text {reg }}=I_{R}-I_{R}^{\text {div }}-I_{R}^{\text {sing }}$. Its imaginary part, from the definition (59) and from the choice of the singular part of the diagram, reads as

$$
\operatorname{Im} I_{R}^{\mathrm{reg}}(k)=-\frac{1}{2} \int \frac{d^{6} p}{(2 \pi)^{6}}\left(\frac{1}{2}+n\left(p_{0}\right)\right) \varrho_{0}(p) \varrho_{0}(k-p)+\frac{1}{2(4 \pi)^{3}} \Theta\left(k^{2}-M^{2}\right)\left(\frac{k^{2}}{6}-m^{2}\right) .
$$


From (59) and (61) one can see that the real part can be obtained from the Kramers-Kronig relation

$$
\operatorname{Re} I_{R}^{\mathrm{reg}}(k)=\int_{-\infty}^{\infty} \frac{d \omega}{\pi} \frac{-\operatorname{Im} I_{R}^{\mathrm{reg}}(\omega, \mathbf{k})}{k_{0}-\omega+i \varepsilon}
$$

This integral is convergent, since $\operatorname{Im} I_{R}^{\mathrm{reg}}(k) \sim 1 / k_{0}^{2}$ for large $k_{0}$ values.

The self-energy reads with these choices as

$$
\Sigma_{R}(k)=-k^{2} \delta Z_{0}^{2}-m^{2} \delta Z_{m}^{2}+g^{2} I_{R}^{\text {sing }}(k)+g^{2} I_{R}^{\mathrm{reg}}(k)+\delta m_{R}^{2}(k)+k^{2} \delta Z^{2} .
$$

In the $2 \mathrm{PI}$ scheme we choose the counterterms in a specific way, namely we choose

$$
\delta m_{R}^{2}(k)=m^{2} \delta Z_{m}^{2}-g^{2} I_{R}^{\mathrm{reg}}(k), \quad \delta Z^{2}=\delta Z_{0}^{2} .
$$

Thus almost all terms are canceled in the self energy; what remains is

$$
\Sigma_{R}^{r e n}(k)=g^{2} I_{R}^{\text {sing }}(k)=\frac{g^{2}}{2(4 \pi)^{3}}\left(\frac{k^{2}}{6}-m^{2}\right) \ln \frac{M^{2}-k^{2}}{\mu^{2}},
$$

where the Landau prescription $\left(k_{0} \rightarrow k_{0}+i \varepsilon\right)$ is implicitly understood in the formula. We can observe that this expression is almost constant in the IR regime where $k^{2} \ll M^{2}$. For convenience, in the numerical calculations we have chosen $\mu=M$, and so this constant is $\sim \ln \left(1-k^{2} / M^{2}\right) \ll 1$ for $k^{2} \ll M^{2}$.

The full retarded propagator in this scheme reads as

$$
G_{R}^{-1}(k)=k^{2}-m_{R}^{2}(k)-\frac{g^{2}}{2(4 \pi)^{3}}\left(\frac{k^{2}}{6}-m^{2}\right) \ln \frac{M^{2}-k^{2}}{\mu^{2}} .
$$

Our reference scheme will be the renormalization scheme defined by the mass counterterm $\delta m_{\text {ref }}^{2}=m^{2} \delta Z_{m}^{2}$ and wave function counterterm $\delta Z_{\text {ref }}^{2}=\delta Z_{0}^{2}$. For comparability of the results we have to ensure that the bare Lagrangian is the same in the resummed and in the reference scheme. Since the wave function renormalization is the same in the two schemes, we have to ensure that

$$
Z^{2} m_{\text {bare }}^{2}=m_{\text {ref }}^{2}+\delta m_{\text {ref }}^{2}=m_{R}^{2}(k)+\delta m_{R}^{2}(k)=m_{R}^{2}(k)+m^{2} \delta Z_{m}^{2}-g^{2} I_{R}^{\text {reg }}(k) .
$$

This equation implies

$$
m_{R}^{2}(k)=m_{\mathrm{ref}}^{2}+g^{2} I_{R}^{\mathrm{reg}}(k) .
$$

This is a finite gap equation that can be solved numerically.

To determine the value of $m_{\text {ref }}^{2}$ we used the renormalization condition that the exact mass should be a predefined value $m^{2}$ at zero temperature; ie. we required

$$
m_{R}^{2}\left(k^{2}=m^{2}, T=0\right)=m^{2} .
$$

From (77) we can read off the value of $m_{\text {ref }}^{2}$, which later can be used at finite temperature calculations.

Before giving the results of this calculation, we mention that formally it is also possible to choose $\mu^{2}=M^{2}-k^{2}$. With this choice the 2PI scheme is exact. The validity of the above formulae can be maintained, if we choose the same scale in the reference scheme. Then the condition of the constant physics is modified as:

$$
m_{R}^{2}(k)=m_{\mathrm{ref}}^{2}(\mu)+g^{2}(\mu) I_{R}^{\mathrm{reg}}(k) .
$$

Here $m_{\text {ref }}^{2}(\mu)$ and $g^{2}(\mu)$ can be explicitly calculated using the standard renormalization group. This choice may be advantageous in case of asymptotically free theories. In the $\Phi^{3}$ model, however, we have to be careful because of the UV Landau pole.

\section{A. Results}

For numerical calculation we used the spatial rotational invariance of the propagators, and so at finite temperature they are two-dimensional functions, with variables $p_{0}$ and $|\mathbf{p}|$. The integrations are performed on $2 \mathrm{D}$ lattices, which are mapped with a continuous function to the infinite $2 \mathrm{D}$ space. 

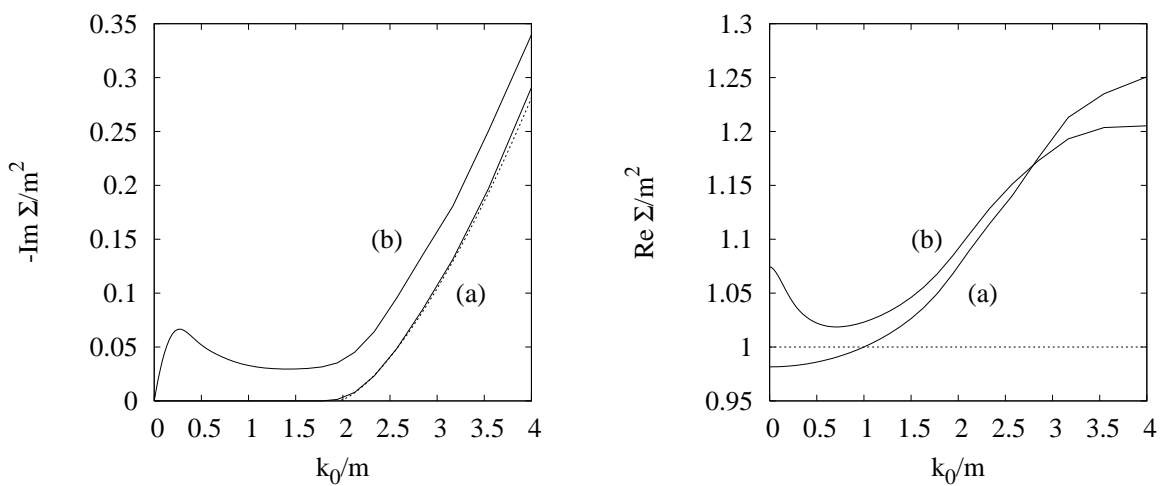

FIG. 1: The imaginary and real part of the retarded self energy at zero temperature (a) and at finite temperature $(T / m=1)$ (b). The spatial momentum is $k / m=0.25$ for the imaginary part case and $k=0$ for the real part.

The main result of the calculation is the retarded self-energy. Its imaginary and real part at zero and at finite temperature can be seen on Fig. 1 In this run the parameters are $g=20, M / m=10$ and for the finite temperature part $T / m=1$. The first plot shows the imaginary part. We can see that at zero temperature (curve (a)) it is zero below the 2-particle threshold $k_{0} / m=2$. With dotted line we plotted the one loop result from (64). We can see that below the 3 -particle threshold $\left(k_{0} / m=3\right)$ the agreement is rather good. The most prominent phenomena of this plot is that at finite temperature the spectral function is non-vanishing everywhere (except zero) - this is what we expect on general arguments. One can observe the smeared-out remnant of the Landau-damping (for $k_{0}<k$ ), but between the Landau-damping region and the zero temperature threshold the spectral function is still large. Remarkably, in this regime the imaginary part is almost constant.

On the second plot of Fig. 1 the real part of the full self energy from the same calculation is depicted. We can see that at zero temperature it is correctly renormalized, we indeed obtain $\Sigma\left(k_{0} / m=1\right)=m^{2}$. At finite temperature we obtained larger real part: this means that the temperature dependent mass correction is positive. In the thermal mass approximation the finite temperature curve would be above the zero temperature part by a constant shift. But, according to this figure, there is considerable difference between the $k_{0}=0$ contribution (Debye-mass) and the $k_{0}=m$ contribution (on-shell mass correction).

On Fig. 2 the temperature dependence of the quasiparticle properties can be seen. In the first plot the thermal
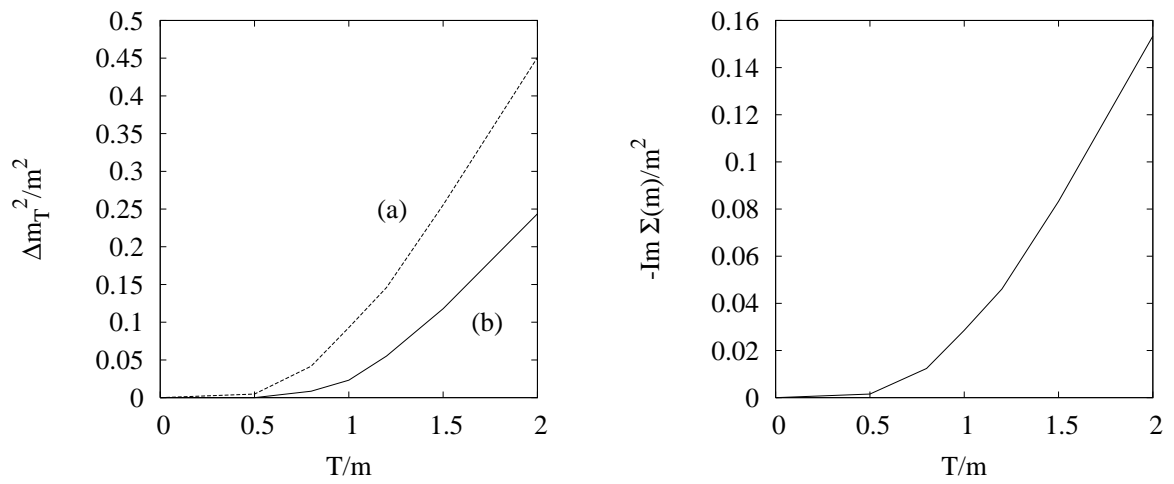

FIG. 2: The first plot describes the thermal mass correction. The dashed curve (a) corresponds to the Debye mass $\left(\right.$ ie. $k_{0}=0$ case), the solid curve (b) is the on-shell value. In the second plot the on-shell imaginary part of the self energy can be seen.

mass correction is shown. The two curves correspond to the thermal mass corrections evaluated at $k_{0}=0$ (curve (a): this is the Debye- or screening mass) and on mass shell (curve (b): this is the quasiparticle mass). There is a rather big difference between the two values, which indicates that a single effective mass term cannot be a satisfactory description. In the regime $T / m>\mathcal{O}(1)$ both corrections can be well fitted by a quadratic function of the temperature $\left(a T^{2}+b\right)$, but in the small temperature regime the curve is much shallower. This means that although the formula $m_{\text {eff }}^{2} \approx \bar{m}^{2}+a T^{2}$ is valid at high temperatures, but $\bar{m}$ is smaller than the zero temperature mass. If this is a robust phenomenon persisting to models with spontaneous symmetry breaking, it should lead to increasing of the critical temperature of a phase transition as compared to the pure perturbation theory. In the current case $(g=20)$ the mass 
modification is about $5 \%$.

In the second plot of Fig. 2 the on-shell value of the imaginary part of the self energy can be seen as a function of the temperature. This is purely resummation effect: at one loop level there is no on-shell damping. The result again fits well to a quadratic temperature curve $\left(a T^{2}+b\right)$ in the regime $T / m>\mathcal{O}(1)$, and it is shallower than quadratic for small temperatures. At high temperatures it leads to a quasiparticle damping which depends linearly on the temperature.

\section{CONCLUSIONS}

When we leave the realm of the strict perturbative calculations and try to implement exact, or approximate resummation formulae (like Schwinger-Dyson equations or 2PI resummation equations), we encounter new type of divergences in the calculation. We have then two ways to proceed. One is that we try to find out the structure of the new type of divergences, then resum the counterterms in the same way, thus rendering the calculation finite. An alternative way is to realize that divergences come solely from the ultraviolet regime which is perturbative (if it is perturbative at zero temperature), while the interesting results are at low energies. This allows to change the resummation equations in a way that they do nothing at high energies, but do resummation in the physically important IR regime.

In this alternative way the divergence structure does not vary with the environment, which means that, in fact, we use a special renormalization scheme. Technically this scheme works in a way that we choose the finite parts of the counterterms (which are free to choose) to cancel the IR part of the IR sensitive diagrams. The remaining perturbation theory is no more IR sensitive, so we can safely use it. The results are, however, obtained in a weird scheme which depends on the environment, while we would need the result in a well defined, fixed scheme (eg. in $\overline{\mathrm{MS}})$. But results calculated in two schemes can always be connected by renormalization group transformation: we only have to ensure that the original bare Lagrangian is the same in the two cases. To satisfy this requirement we have to write up constraint equations which are, in the original resummation language, the gap equations.

This strategy can be used for momentum dependent schemes, too. In particular one can derive a $2 \mathrm{PI}$ scheme where, in the IR regime, all the two particle reducible diagrams are canceled in the perturbation theory. The requirement of keeping the bare Lagrangian constant can be translated to a consistency equation for the momentum dependent mass. If we treat the UV characteristics of the theory well, these equations provide finite solutions.

To demonstrate in an example how this strategy works, we computed the 2PI resummed renormalized self energy in the six dimensional $\Phi^{3}$ model. We used $\overline{\mathrm{MS}}$ scheme as reference scheme, and we studied finite temperature dependence of different physical quantities. The model at relatively large coupling $(g=20)$ still can be characterized by finite lifetime quasiparticles, but the spectral function (density of states) is nonzero for all nonzero frequencies. Interesting result is that the on-shell thermal mass and the zero frequency (Debye) mass are quite different, in the thermal mass correction there is almost a factor of 2 between the two values.

To pursue this project there are two ways open. Since we worked out the technicalities, we will be able to use renormalized 2PI analysis for other models, too. On the other hand, with the same logic we can discuss the 4PI or even higher point irreducible approximations in a renormalized way.

\section{ACKNOWLEDGMENTS}

The author is grateful to A. Arrizabalaga, T.S. Bíró, A. Patkós, J. Polonyi, U. Reinosa and Zs. Szép for useful discussions. This work was supported by Hungarian grant OTKA F043465.

\section{APPENDIX A: THE MASS MATRIX AT FINITE TEMPERATURE}

Most easily we can arrive to the relations between the self-energies in the following way. First we determine the relation between the self energies in the CTP and R/A formalism. For this we write up the quadratic part of the effective Lagrangian in equilibrium:

$$
\mathcal{L}_{\mathrm{eff}}^{(2)}=\frac{1}{2}\left(\Phi^{(1)}, \Phi^{(2)}\right)\left(\begin{array}{cc}
k^{2}-\Sigma_{11} & \Sigma_{12} \\
\Sigma_{21} & k^{2}-\Sigma_{22}
\end{array}\right)\left(\begin{array}{l}
\Phi^{(1)} \\
\Phi^{(2)}
\end{array}\right)
$$


To change to the R/A formalism we use $\Phi^{(1,2)}=\Phi^{r} \pm \frac{1}{2} \Phi^{a}$. Then the effective Lagrangian can be written as

$$
\mathcal{L}_{\text {eff }}^{(2)}=\frac{1}{2}\left(\Phi^{r}, \Phi^{a}\right)\left(\begin{array}{cc}
0 & k^{2}-\Sigma_{r a} \\
k^{2}-\Sigma_{a r} & \Sigma_{a a}
\end{array}\right)\left(\begin{array}{l}
\Phi^{r} \\
\Phi^{a}
\end{array}\right),
$$

where

$$
\begin{aligned}
& \Sigma_{11}+\Sigma_{12}+\Sigma_{21}+\Sigma_{22}=\Sigma_{r r}=0 \\
& \Sigma_{11}+\Sigma_{12}-\Sigma_{21}-\Sigma_{22}=2 \Sigma_{a r} \\
& \Sigma_{11}-\Sigma_{12}+\Sigma_{21}-\Sigma_{22}=2 \Sigma_{r a} \\
& \Sigma_{11}-\Sigma_{12}-\Sigma_{21}+\Sigma_{22}=4 \Sigma_{a a},
\end{aligned}
$$

where we used the information that $G_{a a}=0$, and so $\Sigma_{r r}=0$. The above equations can be written

$$
\begin{aligned}
& \Sigma_{R} \equiv \Sigma_{a r}=\Sigma_{11}+\Sigma_{12} \\
& \Sigma_{A} \equiv \Sigma_{r a}=\Sigma_{11}+\Sigma_{21} \\
& \Sigma_{K} \equiv \Sigma_{a a}=\frac{\Sigma_{21}+\Sigma_{12}}{2}
\end{aligned}
$$

On the other hand, inverting the kernel in the R/A formalism yields the propagators

$$
G_{R}=\frac{1}{k^{2}-\Sigma_{R}}, \quad G_{A}=\frac{1}{k^{2}-\Sigma_{A}}, \quad i G_{K}=\frac{\Sigma_{a a}}{\Sigma_{R}-\Sigma_{A}} \varrho,
$$

where $\varrho=i G_{R}-i G_{A}$. Since $G_{R}^{*}=G_{A}$, therefore $\Sigma_{R}^{*}=\Sigma_{A}$. Moreover, since $i G_{K}=\left(\frac{1}{2}+n\right) \varrho$, so $\Sigma_{a a}=\left(\frac{1}{2}+\right.$ $n)\left(-2 i \operatorname{Im} \Sigma_{R}\right)$. That means

$$
\begin{aligned}
& \Sigma_{11}=\frac{\Sigma_{R}+\Sigma_{A}}{2}-\Sigma_{a a}=\operatorname{Re} \Sigma_{R}+i(1+2 n) \operatorname{Im} \Sigma_{R} \\
& \Sigma_{22}=-\frac{\Sigma_{R}+\Sigma_{A}}{2}+\Sigma_{a a}=-\operatorname{Re} \Sigma_{R}+i(1+2 n) \operatorname{Im} \Sigma_{R} \\
& \Sigma_{12}=\frac{\Sigma_{R}-\Sigma_{A}}{2}+\Sigma_{a a}=-2 i n \operatorname{Im} \Sigma_{R} \\
& \Sigma_{21}=-\frac{\Sigma_{R}-\Sigma_{A}}{2}+\Sigma_{a a}=-2 i(1+n) \operatorname{Im} \Sigma_{R}
\end{aligned}
$$

[1] L. Dolan and R. Jackiw, Phys. Rev. D9 (1974) 3320.

[2] J. Frenkel, A. Saa and J. Taylor, Phys. Rev. D 46 (1992) 3670; R. Parwani, Phys. Rev. D 45 (1992) 4695.

[3] W. Buchmuller, Z. Fodor, T. Helbig and D. Walliser, Ann. Phys. 234 (1994) 260; J.R. Espinosa, M. Quiros and F. Zwirner, Phys. Lett. 314 (1993) 206

[4] F. Karsch, A. Patkos and P. Petreczky, Phys.Lett. B401 (1997) 69-73

[5] E. Braaten and R. D. Pisarski, Nucl. Phys. B 337 (1990) 569. J. Frenkel and J. C. Taylor, Nucl. Phys. B 334 (1990) 199. J. C. Taylor and S. M. H. Wong, Nucl. Phys. B 346 (1990) 115.

[6] J. M. Cornwall,R. Jackiw and E. Toumbolis, Phys. Rev. D10, 2428 (1974).

[7] J. Berges and J. Cox, Phys. Lett. B 517 (2001) 369; A. Arrizabalaga, J. Smit and A. Tranberg, Phys. Rev. D 72 (2005) 025014; G. Aarts and J. Berges, Phys. Rev. D 64 (2001) 105010; J. Berges, Nucl. Phys. A 699 (2002) 847; G. Aarts and J. Berges, Phys. Rev. Lett. 88, 041603 (2002); J. Berges and J. Serreau, Phys. Rev. Lett. 91 (2003) 111601; A. Arrizabalaga, J. Smit and A. Tranberg, JHEP 0410 (2004) 017;

[8] H. C. G. Caldas, A. L. Mota and M. C. Nemes, Phys. Rev. D 63, 056011 (2001); H. C. G. Caldas, Phys. Rev. D 65,065005 (2002)

[9] A. Jakovac and Zs. Szep, Phys. Rev. D 71, 105001 (2005)

[10] N. Banerjee and S. Mallik, Phys. Rev. D 43, 3368 (1991)

[11] H. Verschelde, Phys.Lett. B 497 (2001) 165

[12] D. O'Connor and C.R. Stephens, Int. J. Mod. Phys. A 9 (1994) 2805; Erratum-ibid. A 9 (1994) 5851.

[13] H. van Hees, J. Knoll, Phys. Rev. D65 (2002) 025010; Phys.Rev.D65 (2002) 105005; Phys.Rev.D66 (2002) 025028

[14] J.-P. Blaizot, E. Iancu, U. Reinosa, Phys.Lett.B568 (2003) 160, hep-ph/0301201; Nucl.Phys.A736 (2004) 149, hep-ph/0312085 
[15] J. Berges, Sz. Borsanyi, U. Reinosa and J. Serreau, Annals Phys.320 (2005) 344, hep-ph/0503240

[16] F. Cooper, B. Mihaila and J. F. Dawson, Phys.Rev.D70 (2004) 105008, hep-ph/0407119; U. Reinosa, J. Serreau, hep-th/0605023

[17] C. Destri, A. Sartirana Phys.Rev.D72 (2005) 065003, hep-ph/0504029; Phys.Rev.D73 (2006) 025012, hep-ph/0509032

[18] J. Collins, Renormalization (Cambridge University Press, 1984).

[19] E. Wang and U. Heinz, Phys. Lett. B471, 208 (1999); K.-C. Chou, Z.-B. Su, B.-L. Lao and L. Yu, Phys. Rep. 118, 1 (1985)

[20] R.L Kobes and G.W. Semenoff, Nucl. Phys. B260 (1985) 714.

[21] In gauge theories the introduction of magnetic mass is an example where the effective mass can have an important physical role 